\title{
Existence and Uniqueness of Globally Attractive Positive Almost Periodic Solution in a Predator-Prey Dynamic System with Beddington-DeAngelis Functional Response
}

\author{
Wenquan Wu \\ Department of Mathematics, Aba Teachers College, Wenchuan, Sichuan 623002, China \\ Correspondence should be addressed to Wenquan Wu; mathwqwu@163.com
}

Received 22 January 2014; Accepted 22 July 2014; Published 12 August 2014

Academic Editor: Zhichun Yang

Copyright (C) 2014 Wenquan Wu. This is an open access article distributed under the Creative Commons Attribution License, which permits unrestricted use, distribution, and reproduction in any medium, provided the original work is properly cited.

This paper is concerned with a predator-prey system with Beddington-DeAngelis functional response on time scales. By using the theory of exponential dichotomy on time scales and fixed point theory based on monotone operator, some simple conditions are obtained for the existence of at least one positive (almost) periodic solution of the above system. Further, by means of Lyapunov functional, the global attractivity of the almost periodic solution for the above continuous system is also investigated. The main results in this paper extend, complement, and improve the previously known result. And some examples are given to illustrate the feasibility and effectiveness of the main results.

\section{Introduction}

Let

$$
\begin{gathered}
f^{-}=\inf _{s \in \mathbb{T}} f(s), \quad f^{+}=\sup _{s \in \mathbb{T}} f(s), \\
m(f)=\lim _{l \rightarrow \infty} \frac{1}{l} \int_{0}^{l} f(s) \mathrm{d} s,
\end{gathered}
$$

where $f$ is a continuous bounded function defined on $\mathbb{T}$ and $\mathbb{T}$ is a time scale.

The dynamic relationship between predators and their prey has long been and will continue to be one of the dominant themes in both ecology and mathematical ecology due to its universal existence and importance. One significant component of the predator-prey relationship is the functional responses. In general, the functional responses can be either prey dependent or predator dependent. However, the preydependent ones fail to model the interference among predators and have been facing challenges from the biology and physiology communities. The predator-dependent functional responses can provide better descriptions of predator feeding over a range of predator-prey abundances as is supported by much significant laboratory and field evidence. The Beddington-DeAngelis functional response, first proposed by Beddington [1] and DeAngelis et al. [2], performed even better. So, the dynamics of predator-prey systems with the Beddington-DeAngelis response have been studied extensively in the literature [3-10].

In [8], Cui and Takeuchi considered the following predator-prey system with Beddington-DeAngelis functional response:

$$
\begin{gathered}
x^{\prime}(t)=x(t)[a(t)-b(t) x(t) \\
\left.-\frac{c(t) y(t)}{\alpha(t)+\beta(t) x(t)+\gamma(t) y(t)}\right], \\
y^{\prime}(t)=y(t)\left[-d(t)+\frac{f(t) x(t)}{\alpha(t)+\beta(t) x(t)+\gamma(t) y(t)}\right],
\end{gathered}
$$

where all the coefficients of system (2) are positive $\omega$-periodic functions. Cui and Takeuchi obtained the following. 
Theorem 1 (see [8]). System (2) has at least one positive $\omega$ periodic solution provided

$$
\begin{gathered}
(C) \int_{0}^{\omega}\left[-d(t)+\frac{f x_{0}(t)}{\alpha(t)+\beta(t) x_{0}(t)}\right] \mathrm{d} t>0, \quad \text { where } \\
x_{0}(t)=\frac{1-e^{-\int_{0}^{\omega} a(s) \mathrm{d} s}}{\int_{0}^{\omega} b(t-s) e^{-\int_{0}^{s} a(t-u) \mathrm{d} u} \mathrm{~d} s}
\end{gathered}
$$

In real world phenomenon, the environment varies due to the factors such as seasonal effects of weather, food supplies, mating habits, and harvesting. So it is usual to assume the periodicity of parameters in system (2). However, if the various constituent components of the temporally nonuniform environment are with incommensurable (nonintegral multiples) periods, then one has to consider the environment to be almost periodic since the assumption of almost periodicity is more realistic, more important, and more general when we consider the effects of the environmental factors. However, to the best of the author's knowledge, up to date, there are few works on the existence of positive almost periodic solution of system (2). Therefore, the aim of this paper is to use the fixed point theory based on monotone operator and Lyapunov functional to investigate the positive (almost) periodic solutions of system (2).

In fact, continuous and discrete systems are very important in implementing and applications. It is well known that the theory of time scales has received a lot of attention which was introduced by Hilger [11] in order to unify continuous and discrete analyses. Therefore, it is meaningful to study dynamic systems on time scales which can unify differential and difference systems. Recently, the topic on the dynamics of predator-prey system with Beddington-DeAngelis functional response on time scales has been investigated in some papers (see $[9,10])$. Stimulated by the previous reasons, in this paper we will study the following predator-prey system with Beddington-DeAngelis functional response on time scales:

$$
\begin{gathered}
x^{\Delta}(t)=x(t)[a(t)-b(t) x(t) \\
\left.-\frac{c(t) y(t)}{\alpha(t)+\beta(t) x(t)+\gamma(t) y(t)}\right], \\
y^{\Delta}(t)=y(t)\left[-d(t)+\frac{f(t) x(t)}{\alpha(t)+\beta(t) x(t)+\gamma(t) y(t)}\right],
\end{gathered}
$$

where $t \in \mathbb{T}$ is a periodic time scale; all the coefficients of system (4) are nonnegative almost periodic functions. From the point of view of biology, we focus our discussion on the existence of positive almost periodic solution of system (4) by using the theory of exponential dichotomy on time scales and fixed point theory based on monotone operator. Further, with the help of Lyapunov functional, the global attractivity of a unique positive almost periodic solution of system (2) is considered.

The remainder of this paper is organized in the following ways. In Section 2, we will introduce some necessary notations, definitions, and lemmas which will be used in the paper.
In Section 3, some easy conditions are derived ensuring the existence of at least one positive (almost) periodic solution of system (4) by using the theory of exponential dichotomy on time scales and fixed point theorem of monotone operator. In Section 4, we establish sufficient conditions for the global attractivity of a unique positive (almost) periodic solution of the corresponding continuous system (4) (i.e., system (2)) by means of Lyapunov functional. The main results are illustrated by giving some examples in Section 5 .

\section{Preliminaries}

Now, let us state the following definitions and lemmas, which will be useful in proving our main result.

Definition 2 (see [12]). A time scale $\mathbb{T}$ is an arbitrary nonempty closed subset of the real set $\mathbb{R}$ with the topology and ordering inherited from $\mathbb{R}$. The forward and backward jump operators $\sigma, \rho: \mathbb{T} \rightarrow \mathbb{T}$ and the graininess $\mu, \nu: \mathbb{T} \rightarrow$ $\mathbb{R}^{+}$are defined, respectively, by

$$
\begin{array}{rlrl}
\sigma(t):=\inf \{s \in \mathbb{T}: s>t\}, & & \rho(t):=\sup \{s \in \mathbb{T}: s<t\}, \\
\mu(t):=\sigma(t)-t, & \nu(t):=t-\rho(t) .
\end{array}
$$

The point $t \in \mathbb{T}$ is called left-dense, left-scattered, right-dense, or right-scattered if $\rho(t)=t, \rho(t)<t, \sigma(t)=t$, or $\sigma(t)>t$, respectively. Points that are right-dense and left-dense at the same time are called dense. If $\mathbb{T}$ has a left-scattered maximum $m_{1}$, define $\mathbb{T}^{\mathcal{K}}=\mathbb{T}-\left\{m_{1}\right\}$; otherwise, set $\mathbb{T}^{\mathcal{K}}=\mathbb{T}$. If $\mathbb{T}$ has a right-scattered minimum $m_{2}$, define $\mathbb{T}_{\kappa}=\mathbb{T}-\left\{m_{2}\right\}$; otherwise, set $\mathbb{T}_{\kappa}=\mathbb{T}$.

Definition 3 (see [12]). A function $p: \mathbb{T} \rightarrow \mathbb{R}$ is said to be regressive provided $1+\mu(t) p(t) \neq 0$ for all $t \in \mathbb{T}^{k}$, where $\mu(t)=$ $\sigma(t)-t$ is the graininess function. The set of all regressive rdcontinuous functions $f: \mathbb{T} \rightarrow \mathbb{R}$ is denoted by $\mathscr{R}$ while the set $\mathscr{R}^{+}$is given by $\{f \in \mathscr{R}: 1+\mu(t) f(t)>0\}$ for all $t \in \mathbb{T}$. Let $p \in \mathscr{R}$. The exponential function is defined by

$$
e_{p}(t, s)=\exp \left(\int_{s}^{t} \xi_{\mu(\tau)}(p(\tau)) \Delta \tau\right),
$$

where $\xi_{h(z)}$ is the so-called cylinder transformation.

Lemma 4 (see [12]). Let $p, q \in \mathscr{R}$. Then

(i) $e_{0}(t, s) \equiv 1$ and $e_{p}(t, t) \equiv 1$;

(ii) $1 / e_{p}(t, s)=e_{\ominus p}(t, s)$, where $\ominus p(t)=-p(t) /(1+$ $\mu(t) p(t))$

(iii) $e_{p}(t, s) e_{p}(s, r)=e_{p}(t, r)$;

(iv) $e_{p}^{\Delta}(\cdot, s)=p e_{p}(\cdot, s)$.

Definition 5 (see [12]). For $f: \mathbb{T} \rightarrow \mathbb{R}$ and $t \in \mathbb{T}^{k}$, the delta derivative of $f$ at $t$, denoted by $f^{\Delta}(t)$, is the number (provided 
it exists) with the property that, given any $\epsilon>0$, there is a neighborhood $U \subset \mathbb{T}$ of $t$ such that

$$
\begin{array}{r}
\left|f(\sigma(t))-f(s)-f^{\Delta}(t)[\sigma(t)-t]\right| \leq \epsilon|\sigma(t)-s|, \\
\forall s \in U .
\end{array}
$$

Lemma 6 (see [12]). Assume that $p(t) \geq 0$ for $t \geq 0$. Then $e_{p}(t, s) \geq 1$.

Lemma 7 (see [12]). Suppose that $p \in \mathscr{R}^{+}$. Then

(i) $e_{p}(t, s)>0$ for all $t, s \in \mathbb{T}$;

(ii) if $p(t) \leq q(t)$ for all $t \geq s, t, s \in \mathbb{T}$, then $e_{p}(t, s) \leq$ $e_{q}(t, s)$ for all $t \geq s$.

Lemma 8 (see [12]). Suppose that $p \in \mathscr{R}$ and $a, b, c \in \mathbb{T}$; then

$$
\begin{gathered}
{\left[e_{p}(c, \cdot)\right]^{\Delta}=-p\left[e_{p}(c, \cdot)\right]^{\sigma},} \\
\int_{a}^{b} p(t) e_{p}(c, \sigma(t)) \Delta t=e_{p}(c, a)-e_{p}(c, b) .
\end{gathered}
$$

Definition 9 (see [13]). A time scale $\mathbb{T}$ is called a periodic time scale if

$$
\Pi:=\{\tau \in \mathbb{R}: t+\tau \in \mathbb{T}, \forall t \in \mathbb{T}\} \neq\{0\} .
$$

Definition 10 (see [14]). Let $\mathbb{T}$ be a periodic time scale. A function $x: \mathbb{T} \rightarrow \mathbb{R}^{n}$ is called almost periodic on $\mathbb{T}$, if, for any $\epsilon>0$, the set

$$
E(\epsilon, x)=\{\tau \in \Pi:|x(t+\tau)-x(t)|<\epsilon, \forall t \in \mathbb{T}\}
$$

is relatively dense in $\mathbb{\mathbb { T }}$; that is, there exists a constant $l=l(\epsilon)>$ 0 , for any interval with length $l(\epsilon)$; there exists a number $\tau=$ $\tau(\epsilon)$ in this interval such that

$$
\|x(t+\tau)-x(t)\|<\epsilon, \quad \forall t \in \mathbb{T} .
$$

The set $E(\epsilon, x)$ is called the $\epsilon$-translation set of $x ; \tau$ is called the $\epsilon$-translation number of $x$, and $l(\epsilon)$ is called the inclusion of $E(\epsilon, x)$.

Definition 11 (see [15]). Let $y \in C\left(\mathbb{T}, \mathbb{R}^{n}\right)$ and let $P(t)$ be $n \times n$ continuous matrix defined on $\mathbb{T}$. The linear system

$$
y^{\Delta}(t)=P(t) y(t), \quad t \in \mathbb{T},
$$

is said to be an exponential dichotomy on $\mathbb{T}$ if there exist constants $k, \lambda>0$, projection $S$, and the fundamental matrix $Y(t)$ satisfying

$$
\begin{gathered}
\left\|Y(t) S Y^{-1}(s)\right\| \leq k e_{\ominus \lambda}(t, s), \quad \forall t \geq s, \\
\left\|Y(t)(I-S) Y^{-1}(s)\right\| \leq k e_{\ominus \lambda}(s, t), \quad \forall t \leq s, t, s \in \mathbb{T} .
\end{gathered}
$$

Lemma 12 (see [16]). If the linear system $y^{\Delta}(t)=P(t) y(t)$ has an exponential dichotomy, then almost periodic system

$$
y^{\Delta}(t)=P(t) y(t)+g(t), \quad t \in \mathbb{T},
$$

has a unique almost periodic solution $y(t)$ which can be expressed as follows:

$$
\begin{aligned}
y(t)= & \int_{-\infty}^{t} Y(t) S Y^{-1}(\sigma(s)) g(s) \Delta s \\
& -\int_{t}^{\infty} Y(t)(I-S) Y^{-1}(\sigma(s)) g(s) \Delta s .
\end{aligned}
$$

Lemma 13 (see [15]). If $P(t)=\left(a_{i j}(t)\right)_{n \times n}$ is a uniformly bounded rd-continuous matrix-valued function on $\mathbb{T}$ and there is a $\delta>0$ such that

$$
\begin{array}{r}
\left|a_{i i}(t)\right|-\sum_{j \neq i}\left|a_{i j}(t)\right|-\frac{1}{2} \mu(t)\left[\sum_{j \neq i}\left|a_{i j}(t)\right|\right]^{2}-\delta^{2} \mu(t) \geq 2 \delta, \\
t \in \mathbb{T}, \quad i=1,2, \ldots, n,
\end{array}
$$

then $y^{\Delta}(t)=P(t) y(t)$ admits an exponential dichotomy on $\mathbb{T}$.

Lemma 14 (see [12]). Suppose that $r: \mathbb{T} \rightarrow \mathbb{R}$ is regressive. Let $t_{0} \in \mathbb{T}$ and $y_{0} \in \mathbb{R}$. The unique solution of the initial value problem

$$
y^{\Delta}(t)=r(t) y(t)+g(t), \quad y\left(t_{0}\right)=y_{0}
$$

is given by

$$
y(t)=e_{r}\left(t, t_{0}\right) y_{0}+\int_{t_{0}}^{t} e_{r}(t, \sigma(s)) g(s) \Delta s .
$$

Similar to the proof as that in $[14,16]$, we can easily obtain from Lemmas 12-14 the following.

Lemma 15. Assume that $\left(H_{1}\right)-\left(H_{2}\right)$ hold; then system (4) has a unique almost periodic solution $z=(x, y)^{T}$ which can be expressed as follows:

$$
\begin{aligned}
& x(t)=\int_{t}^{+\infty} e_{a}(t, \sigma(s)) x(s) \\
& \times {\left[b(s) x(s)+\frac{c(s) y(s)}{\alpha(s)+\beta(s) x(s)+\gamma(s) y(s)}\right] \Delta s, } \\
& y(t)=\int_{-\infty}^{t} \frac{e_{-d}(t, \sigma(s)) f(s) x(s) y(s)}{\alpha(s)+\beta(s) x(s)+\gamma(s) y(s)} \Delta s .
\end{aligned}
$$

In order to obtain the existence of positive almost periodic solution of system (4), we first make the following preparations.

Let $E$ be a Banach space and let $K$ be a cone in $E$. The semiorder induced by the cone $K$ is denoted by " $\leq$ ". That is, $x \leq y$ if and only if $y-x \in K . x<y$ if $x \leq y$ and $x \neq y$. $x \gg y$ if $x-y \in \widehat{K}$, where $\widehat{K}$ is the interior of the cone $K$. A cone $K$ is called minihedral if, for any pair $\{x, y\}, x, y \in$ $E$, bounded above in order that there exists the least upper bound $\sup \{x, y\}$. A cone $K$ is called normal if there exists a constant $N>0$ such that $x \leq y, x, y \in K$ implies $\|x\|_{E} \leq$ $N\|y\|_{E}$. 
Definition 16 (see [17]). $\Phi: K \rightarrow K$ is said to be monotone increasing, if, for $\forall x_{1}, x_{2} \in K, x_{1} \leq x_{2}$, one has $\Phi x_{1} \leq \Phi x_{2}$.

The following two lemmas cited from [18] are useful for the proof of our main results in this section.

Lemma 17 (see [18]). Let $E$ be a real Banach space with an order cone $K$ satisfying the following:

(a) K has a nonempty interior,

(b) $K$ is normal and minihedral.

Assume that there are two points in $E, x_{*} \ll x^{*}$, and a monotone increasing complete continuous operator $\Phi:\left[x_{*}, x^{*}\right] \rightarrow$ E. If

$$
\Phi x_{*} \ll x_{*}, \quad x^{*} \ll \Phi x^{*},
$$

then $\Phi$ has a fixed point $x \in\left[x_{*}, x^{*}\right]$. Here $\left[x_{*}, x^{*}\right]$ denotes the order interval $\left\{x \in K: x_{*} \leq x \leq x^{*}\right\}$.

Consider the Banach space $E=\operatorname{AP}\left(\mathbb{T}, \mathbb{R}^{n}\right)$ with the norm

$$
\|x\|=\max \left\{x^{+}, y^{+}\right\}, \quad \forall z=(x, y)^{T} \in E .
$$

Define the cone $K$ in $E$ by

$$
K=\left\{z=(x, y)^{T} \in E: x \geq 0, y \geq 0\right\} .
$$

It is not difficult to verify that $K$ is normal and minihedral and has a nonempty interior.

Let the map $L$ be defined by

$$
(L z)(t)=((\Phi z)(t),(\Psi z)(t))^{T},
$$

where

$$
\begin{aligned}
& (\Phi z)(t) \\
& =\int_{t}^{+\infty} e_{a}(t, \sigma(s)) x(s) \\
& \quad \times\left[b(s) x(s)+\frac{c(s) y(s)}{\alpha(s)+\beta(s) x(s)+\gamma(s) y(s)}\right] \Delta s \\
& (\Psi z)(t)=\int_{-\infty}^{t} \frac{e_{-d}(t, \sigma(s)) f(s) x(s) y(s)}{\alpha(s)+\beta(s) x(s)+\gamma(s) y(s)} \Delta s
\end{aligned}
$$

where $z \in K, t \in \mathbb{T}$.

By $\left(H_{1}\right)-\left(H_{2}\right)$, one could choose some positive constants $x_{*}<x^{*}$ and $y_{*}<y^{*}$ satisfying

$$
\begin{gathered}
{\left[b^{+} x_{*}+\frac{c^{+} y_{*}}{\alpha^{-}+\beta^{-} x_{*}+\gamma^{-} y_{*}}\right]<a^{-},} \\
\frac{f^{+} x_{*}}{\alpha^{-}+\beta^{-} x_{*}+\gamma^{-} y_{*}}<d^{-}, \\
{\left[b^{-} x^{*}+\frac{c^{-} y^{*}}{\alpha^{+}+\beta^{+} x^{*}+\gamma^{+} y^{*}}\right]>a^{+},} \\
\frac{f^{-} x^{*}}{\alpha^{+}+\beta^{+} x^{*}+\gamma^{+} y^{*}}>d^{+} .
\end{gathered}
$$

Lemma 18. $L: D \rightarrow E$ is monotone increasing, where $D=$ $\left[z_{*}, z^{*}\right], z_{*}=\left(x_{*}, y_{*}\right)^{T}$, and $z^{*}=\left(x^{*}, y^{*}\right)^{T}$.

Proof. Let $F_{1}(t, x, y)=x[b(t) x+(c(t) y /(\alpha(t)+\beta(t) x+\gamma(t) y))]$ and $F_{2}(t, x, y)=(f(t) x y /(\alpha(t)+\beta(t) x+\gamma(t) y)), \forall t \in \mathbb{T}$. Then

$$
\begin{aligned}
& (\Phi z)(t)=\int_{t}^{+\infty} e_{a}(t, \sigma(s)) F_{1}(s, x, y) \Delta s \\
& (\Psi z)(t)=\int_{-\infty}^{t} e_{-d}(t, \sigma(s)) F_{2}(s, x, y) \Delta s
\end{aligned}
$$

Notice that

$$
\begin{gathered}
\frac{\partial F_{1}}{\partial x}=2 b(t) x+\frac{c(t) y[\alpha(t)+\gamma(t) y]}{[\alpha(t)+\beta(t) x+\gamma(t) y]^{2}} \geq 0, \\
\frac{\partial F_{1}}{\partial y}=\frac{c(t) x[\alpha(t)+\beta(t) x]}{[\alpha(t)+\beta(t) x+\gamma(t) y]^{2}} \geq 0, \\
\frac{\partial F_{2}}{\partial x}=\frac{f(t) y[\alpha(t)+\gamma(t) y]}{[\alpha(t)+\beta(t) x+\gamma(t) y]^{2}} \geq 0, \\
\frac{\partial F_{2}}{\partial y}=\frac{f(t) x[\alpha(t)+\beta(t) x]}{[\alpha(t)+\beta(t) x+\gamma(t) y]^{2}} \geq 0,
\end{gathered}
$$

which implies that $(L z)(t)=((\Phi z)(t),(\Psi z)(t))^{T}$ is monotone increasing. This completes the proof.

Lemma 19. $\Phi: D \rightarrow E$ is complete continuous.

Proof. First, we show that $L$ maps bounded set into bounded sets. For $\forall z \in D$, we have

$$
\begin{aligned}
\sup _{t \in \mathbb{T}}(\Phi z)(t) \leq & x^{*}\left[b^{+} x^{*}+\frac{c^{+} y^{*}}{\alpha^{-}+\beta^{-} x_{*}+\gamma^{-} y_{*}}\right] \\
& \times \sup _{t \in \mathbb{\mathbb { T }}} \int_{t}^{+\infty} e_{a^{-}}(t, \sigma(s)) \Delta s \\
\leq & \frac{1}{a^{-}} x^{*}\left[b^{+} x^{*}+\frac{c^{+} y^{*}}{\alpha^{-}+\beta^{-} x_{*}+\gamma^{-} y_{*}}\right], \\
\sup _{t \in \mathbb{T}}(\Psi z)(t) \leq & \frac{f^{+} x^{*} y^{*}}{\alpha^{-}+\beta^{-} x_{*}+\gamma^{-} y_{*}} \sup \int_{-\infty}^{t} e_{-d^{-}}(t, \sigma(s)) \Delta s \\
\leq & \frac{1}{d^{-}} \frac{f^{+} x^{*} y^{*}}{\alpha^{-}+\beta^{-} x_{*}+\gamma^{-} y_{*}} .
\end{aligned}
$$


That is, $L D$ is uniformly bounded. In addition, for $\forall t_{1}, t_{2} \in \mathbb{T}$ and $t_{1} \leq t_{2}$, notice that

$$
\begin{aligned}
& \left|(\Phi z)\left(t_{1}\right)-(\Phi z)\left(t_{2}\right)\right| \\
& =\mid \int_{t_{1}}^{+\infty} e_{a}\left(t_{1}, \sigma(s)\right) x(s) \\
& \times\left[b(s) x(s)+\frac{c(s) y(s)}{\alpha(s)+\beta(s) x(s)+\gamma(s) y(s)}\right] \Delta s \\
& -\int_{t_{2}}^{+\infty} e_{a}\left(t_{1}, \sigma(s)\right) x(s) \\
& \times\left[b(s) x(s)+\frac{c(s) y(s)}{\alpha(s)+\beta(s) x(s)+\gamma(s) y(s)}\right] \Delta s \\
& +\int_{t_{2}}^{+\infty} e_{a}\left(t_{1}, \sigma(s)\right) x(s) \\
& \times\left[b(s) x(s)+\frac{c(s) y(s)}{\alpha(s)+\beta(s) x(s)+\gamma(s) y(s)}\right] \Delta s \\
& -\int_{t_{2}}^{+\infty} e_{a}\left(t_{2}, \sigma(s)\right) x(s) \\
& \times\left[b(s) x(s)+\frac{c(s) y(s)}{\alpha(s)+\beta(s) x(s)+\gamma(s) y(s)}\right] \Delta s \mid \\
& \leq \mid \int_{t_{1}}^{t_{2}} e_{a}\left(t_{1}, \sigma(s)\right) x(s) \\
& \times\left[b(s) x(s)+\frac{c(s) y(s)}{\alpha(s)+\beta(s) x(s)+\gamma(s) y(s)}\right] \Delta s \mid \\
& +\mid \int_{t_{2}}^{+\infty}\left[e_{a}\left(t_{2}, \sigma(s)\right)-e_{a}\left(t_{1}, \sigma(s)\right)\right] x(s) \\
& \times\left[b(s) x(s)+\frac{c(s) y(s)}{\alpha(s)+\beta(s) x(s)+\gamma(s) y(s)}\right] \Delta s \mid \\
& \leq x^{*}\left[b^{+} x^{*}+\frac{c^{+} y^{*}}{\alpha^{-}+\beta^{-} x_{*}+\gamma^{-} y_{*}}\right]\left|t_{2}-t_{1}\right| \\
& +\frac{x^{*}}{a^{-}}\left[b^{+} x^{*}+\frac{c^{+} y^{*}}{\alpha^{-}+\beta^{-} x_{*}+\gamma^{-} y_{*}}\right] \\
& \times\left|1-e_{a^{+}}\left(t_{1}, t_{2}\right)\right| \longrightarrow 0, \quad \text { as } t_{1} \longrightarrow t_{2} \text {. }
\end{aligned}
$$

Similarly, one could easily obtain that

$$
\left|(\Psi z)\left(t_{1}\right)-(\Psi z)\left(t_{2}\right)\right| \longrightarrow 0, \quad \text { as } \quad t_{1} \longrightarrow t_{2} .
$$

So $L z$ is equicontinuous for any $z \in D$. Using Arzela-Ascoli theorem on time scales [19], we obtain that $L D$ is relatively compact. In view of Lebesgue's dominated convergence theorem on time scales [20], it is easy to prove that $L$ is continuous. Hence, $L$ is complete continuous. The proof of this lemma is complete.

\section{Almost Periodic Solution}

In this section, we will utilize Lemma 17 which is given in the previous section to establish some sufficient criteria for the existence of positive (almost) periodic solutions of system (4).

Theorem 20. Assume that the following conditions hold:

$$
\begin{aligned}
& \left(H_{1}\right) f^{-}>\beta^{+} d^{+}, \\
& \left(H_{2}\right) a^{-}>0, d^{-}>0, \text { and } \alpha^{-}>0 .
\end{aligned}
$$

Then system (4) has at least one positive almost periodic solution.

Proof. Now, we should use Lemma 17 to prove the existence of positive almost periodic solutions of system (4). By Lemmas 18 and 19, we know that $L$ is a monotone increasing complete continuous operator on $D$. It remains to prove that

$$
L z_{*} \ll z_{*}, \quad z^{*} \ll L z^{*} .
$$

On the one hand, by the definition of $z_{*}=\left(x_{*}, y_{*}\right)^{T}$, it follows that

$$
\begin{aligned}
\Phi z_{*}= & \Phi\left(x_{*}, y_{*}\right)^{T} \\
= & \int_{t}^{\infty} e_{a}(t, \sigma(s)) x_{*} \\
& \times\left[b(s) x_{*}+\frac{c(s) y_{*}}{\alpha(s)+\beta(s) x_{*}+\gamma(s) y_{*}}\right] \Delta s \\
\leq & \frac{1}{a^{-}} x_{*}\left[b^{+} x_{*}+\frac{c^{+} y_{*}}{\alpha^{-}+\beta^{-} x_{*}+\gamma^{-} y_{*}}\right] \\
< & x_{*}, \\
\Psi z_{*}= & \Psi\left(x_{*}, y_{*}\right)^{T} \\
= & \int_{-\infty}^{t} \frac{e_{-d}(t, \sigma(s)) f(s) x_{*} y_{*}}{\alpha(s)+\beta(s) x_{*}+\gamma(s) y_{*}} \Delta s \\
\leq & \frac{1}{d^{-}} \frac{f^{+} x_{*} y_{*}}{\alpha^{-}+\beta^{-} x_{*}+\gamma^{-} y_{*}} \\
< & y_{*},
\end{aligned}
$$

which implies that

$$
L z_{*}=\left(\Phi z_{*}, \Psi z_{*}\right)^{T}<\left(x_{*}, y_{*}\right)^{T}=z_{*} \Longrightarrow L z_{*} \ll z_{*} .
$$

On the other hand, one has from the definition of $z^{*}=$ $\left(x^{*}, y^{*}\right)^{T}$ that

$$
\begin{aligned}
\Phi z^{*} & =\Phi\left(x^{*}, y^{*}\right)^{T} \\
& =\int_{t}^{\infty} e_{a}(t, \sigma(s)) x^{*}
\end{aligned}
$$




$$
\begin{aligned}
& \times\left[b(s) x^{*}+\frac{c(s) y^{*}}{\alpha(s)+\beta(s) x^{*}+\gamma(s) y^{*}}\right] \Delta s \\
\geq & \frac{1}{a^{+}} x^{*}\left[b^{-} x^{*}+\frac{c^{-} y^{*}}{\alpha^{+}+\beta^{+} x^{*}+\gamma^{+} y^{*}}\right] \\
> & x^{*}, \\
\Psi z^{*}= & \Psi\left(x^{*}, y^{*}\right)^{T} \\
= & \int_{-\infty}^{t} \frac{e_{-d}(t, \sigma(s)) f(s) x^{*} y^{*}}{\alpha(s)+\beta(s) x^{*}+\gamma(s) y^{*}} \Delta s \\
\geq & \frac{1}{d^{+}} \frac{f^{-} x^{*} y^{*}}{\alpha^{+}+\beta^{+} x^{*}+\gamma^{+} y^{*}} \\
> & y^{*},
\end{aligned}
$$

which implies that

$$
L z^{*}=\left(\Phi z^{*}, \Psi z^{*}\right)^{T}>\left(x^{*}, y^{*}\right)^{T}=z^{*} \Longrightarrow L z^{*} \gg z^{*} .
$$

Applying Lemma 17, we see that $L$ has at least one positive fixed point in $\left[z_{*}, z^{*}\right]$. Therefore, system (4) has at least one positive almost periodic solution. This completes the proof.

From Theorem 20, we can easily obtain the following.

Theorem 21. Assume that $\left(H_{1}\right)-\left(H_{2}\right)$ hold. Suppose further that all the coefficients of system (4) are nonnegative $\omega$-periodic functions; then system (4) has at least one positive $\omega$-periodic solution.

If $\mathbb{T}=\mathbb{R}$ in system (4), then Theorem 21 is changed to the following theorem.

Theorem 22. Assume that $\left(H_{1}\right)-\left(H_{2}\right)$ hold. Suppose further that all the coefficients of system (2) are nonnegative $\omega$-periodic functions; then system (2) has at least one positive $\omega$-periodic solution.

Remark 23. Clearly, the validity of condition $(C)$ in Theorem 1 depends on coefficients $a, b, d, f, \alpha$, and $\beta$ of system (2). But condition $\left(H_{1}\right)$ in Theorem 22 only depends on coefficients $d, f$, and $\beta$. Therefore, compared with Theorem 1, Theorem 22 is easy to verify and then has an extensive application. Sometimes one cannot judge the existence of the periodic solution for some system in the form of (2) by Theorem 1. However, it can be done by the result in the present theorem. The following example is given to illustrate this point in detail.

Example 24. Let $a(t)=0.1, b(t)=2, c(t)=2, d(t)=(1+$ $1 / 2 \sin t) / 10, f(t)=2, \alpha(t)=3+\sin t, \beta(t)=8+\sin t$, and $\gamma(t)=2+\cos t$; then system (2) becomes

$x^{\prime}(t)$

$$
=x(t)[0.1-2 x(t)
$$

$$
\left.-\frac{2 y(t)}{3+\sin t+(8+\sin t) x(t)+(2+\cos t) y(t)}\right] \text {, }
$$

$y^{\prime}(t)$

$$
\begin{aligned}
=y(t)[ & -\frac{1+1 / 2 \sin t}{10} \\
& \left.+\frac{2 x(t)}{3+\sin t+(8+\sin t) x(t)+(2+\cos t) y(t)}\right] .
\end{aligned}
$$

We have $f^{-}=2>9 \times 0.15=\beta^{+} d^{+}$, which implies from Theorem 22 that system (36) has at least one positive $2 \pi$ periodic solution.

However, the assumption of Theorem 1 does not hold for system (36) because $x_{0} \equiv 0.05$ and

$$
\begin{aligned}
-d(t)+\frac{f x_{0}(t)}{\alpha(t)+\beta(t) x_{0}(t)} & \leq-0.05+\frac{2 \times 0.05}{2+7 \times 0.05} \\
& \approx-0.0074<0 .
\end{aligned}
$$

Therefore one cannot judge the existence of positive periodic solution of system (36) by Theorem 1 .

\section{Global Attractivity}

In this section, we will construct a suitable Lyapunov functional to establish some sufficient criteria for the global attractivity of a unique positive (almost) periodic solution of system (2).

Theorem 25. Assume that $\left(H_{1}\right)-\left(H_{2}\right)$ hold; suppose further that there exists a constant $\rho>0$ such that

$$
\begin{gathered}
\inf _{t \in \mathbb{R}}\left[b(t)-\frac{\alpha(t) f(t)+f(t) \gamma(t) y^{*}+c(t) \beta(t) y^{*}}{\alpha(t)\left[\alpha(t)+\beta(t) x_{*}+\gamma(t) y_{*}\right]}\right]>\rho, \\
\inf _{t \in \mathbb{R}}\left[\frac{\gamma(t) f(t) x_{*}}{\alpha(t)\left[\alpha(t)+\beta(t) x_{*}+\gamma(t) y^{*}\right]}\right. \\
\left.-\frac{\alpha(t) c(t)+c(t) \beta(t) x^{*}}{\alpha(t)\left[\alpha(t)+\beta(t) x_{*}+\gamma(t) y_{*}\right]}\right]>\rho,
\end{gathered}
$$

where $x_{*}, x^{*}, y_{*}$, and $y^{*}$ are defined as those in Theorem 20. Then system (2) has a unique positive almost periodic solution, which is globally attractive.

Proof. By Theorem 20, system (2) has a unique positive almost periodic solution $(x, y)^{T}$ satisfying

$$
x_{*} \leq x(t) \leq x^{*}, \quad y_{*} \leq y(t) \leq y^{*}, \quad \forall t \in \mathbb{R} .
$$

Suppose that $(u, v)^{T}$ is another positive solution of system (2). Define

$$
V(t)=|\ln x(t)-\ln u(t)|+|\ln y(t)-\ln v(t)|, \quad \forall t \in \mathbb{R} .
$$


Calculating the upper right derivatives of $V$ along the solution of system (2), it follows that

$$
\begin{aligned}
& D^{+} V(t) \\
& =\operatorname{sgn}[x(t)-u(t)]\left[\frac{x^{\prime}(t)}{x(t)}-\frac{u^{\prime}(t)}{u(t)}\right] \\
& +\operatorname{sgn}[y(t)-v(t)]\left[\frac{y^{\prime}(t)}{y(t)}-\frac{v^{\prime}(t)}{v(t)}\right] \\
& =\operatorname{sgn}[x(t)-u(t)] \\
& \times\left(-b(t)[x(t)-u(t)]-\frac{c(t) y(t)}{\alpha(t)+\beta(t) x(t)+\gamma(t) y(t)}\right. \\
& \left.+\frac{c(t) v(t)}{\alpha(t)+\beta(t) u(t)+\gamma(t) v(t)}\right) \\
& +\operatorname{sgn}[y(t)-v(t)] \\
& \times\left[\frac{f(t) x(t)}{\alpha(t)+\beta(t) x(t)+\gamma(t) y(t)}\right. \\
& \left.-\frac{f(t) u(t)}{\alpha(t)+\beta(t) u(t)+\gamma(t) v(t)}\right] \\
& \leq-\left(b(t)-\frac{\alpha(t) f(t)+f(t) \gamma(t) y^{*}+c(t) \beta(t) y^{*}}{\alpha(t)\left[\alpha(t)+\beta(t) x_{*}+\gamma(t) y_{*}\right]}\right) \\
& \times|x(t)-u(t)| \\
& -\left(\frac{\gamma(t) f(t) x_{*}}{\alpha(t)\left[\alpha(t)+\beta(t) x_{*}+\gamma(t) y^{*}\right]}\right. \\
& \left.-\frac{\alpha(t) c(t)+c(t) \beta(t) x^{*}}{\alpha(t)\left[\alpha(t)+\beta(t) x_{*}+\gamma(t) y_{*}\right]}\right)|y(t)-v(t)| \\
& \leq-\rho[|x(t)-u(t)|+|y(t)-v(t)|] .
\end{aligned}
$$

Therefore, $V$ is nonincreasing. Integrating (41) from 0 to $t$ leads to

$$
\begin{array}{r}
V(t)+\rho \int_{0}^{t}[|x(s)-u(s)|+|y(s)-v(s)|] \mathrm{d} s \leq V(0)<+\infty, \\
t \in[0, \infty] .
\end{array}
$$

So

$$
\int_{0}^{\infty}[|x(s)-u(s)|+|y(s)-v(s)|] \mathrm{d} s<+\infty
$$

which implies that

$$
\lim _{s \rightarrow+\infty}|x(s)-u(s)|=\lim _{s \rightarrow+\infty}|y(s)-v(s)|=0 .
$$

Thus, the almost periodic solution of system (2) is globally attractive. The global attractivity implies that the almost periodic solution is unique. This completes the proof.
From Theorem 25, we can easily obtain the following.

Theorem 26. Assume that all the conditions of Theorem 25 hold. Suppose further that all the coefficients of system (2) are nonnegative w-periodic functions; then system (2) has a unique positive $\omega$-periodic solution, which is globally attractive.

\section{Two Examples}

Example 27. Consider the following almost periodic predator-prey system with Beddington-DeAngelis functional response on time scales:

$$
\begin{aligned}
& x^{\Delta}(t) \\
& =x(t) \\
& \times[0.1-2 x(t) \\
& \left.\quad-\frac{2 y(t)}{3+\sin t+(8+\sin (\sqrt{2} t)) x(t)+(2+\cos t) y(t)}\right], \\
& y^{\Delta}(t) \quad y(t) \quad\left[-\frac{1+1 / 2 \sin (\sqrt{3} t)}{10}\right. \\
& \quad \times\left[\frac{2 x(t)}{3+\sin t+(8+\sin (\sqrt{2} t)) x(t)+(2+\cos t) y(t)}\right] .
\end{aligned}
$$

Similar to the argument as that in Example 24, system (45) has at least one positive almost periodic solution by Theorem 20.

Example 28. Consider the following almost periodic predator-prey system with Beddington-DeAngelis functional response:

$$
\begin{aligned}
& x^{\prime}(t) \\
& =x(t) \\
& \times[1-5 x(t) \\
& \left.\quad-\frac{10^{-3} y(t)}{3+\sin (\sqrt{3} t)+0.1 x(t)+(2+\cos (\sqrt{2} t)) y(t)}\right],
\end{aligned}
$$




$$
\begin{aligned}
& y^{\prime}(t) \\
& =y(t) \\
& \quad \times\left[-5+\frac{2 x(t)}{3+\sin (\sqrt{3} t)+0.1 x(t)+(2+\cos (\sqrt{2} t)) y(t)}\right] .
\end{aligned}
$$

Then system (46) has a unique positive almost periodic solution, which is globally attractive.

Proof. Corresponding to system (2), we have $a^{-}=a^{+}=1$, $b^{-}=b^{+}=5, d^{-}=d^{+}=5, c^{-}=c^{+}=10^{-3}, f^{-}=f^{+}=$ $2, \beta^{-}=\beta^{+}=0.1, \alpha^{-}=2, \alpha^{+}=4, \gamma^{-}=1$, and $\gamma^{+}=3$. Obviously, $f^{-}>\beta^{+} d^{+}$. By Theorem 20, system (46) has at least one positive almost periodic solution. Further, we choose $x_{*}=y_{*}=0.1, x^{*}=20$, and $y^{*}=0.3$; then

$$
\begin{gathered}
{\left[b^{+} x_{*}+\frac{c^{+} y_{*}}{\alpha^{-}+\beta^{-} x_{*}+\gamma^{-} y_{*}}\right]<0.8<1=a^{-},} \\
\frac{f^{+} x_{*}}{\alpha^{-}+\beta^{-} x_{*}+\gamma^{-} y_{*}}<0.1<5=d^{-}, \\
{\left[b^{-} x^{*}+\frac{c^{-} y^{*}}{\alpha^{+}+\beta^{+} x^{*}+\gamma^{+} y^{*}}\right]>5>1=a^{+},} \\
\frac{f^{-} x^{*}}{\alpha^{+}+\beta^{+} x^{*}+\gamma^{+} y^{*}}>\frac{40}{7}>5=d^{+},
\end{gathered}
$$

which implies that (25) hold. And

$$
\begin{aligned}
& \inf _{t \in \mathbb{R}} {\left[b(t)-\frac{\alpha(t) f(t)+f(t) \gamma(t) y^{*}+c(t) \beta(t) y^{*}}{\alpha(t)\left[\alpha(t)+\beta(t) x_{*}+\gamma(t) y_{*}\right]}\right] } \\
&> 5-2.5=2.5 \\
& \inf _{t \in \mathbb{R}}\left[\frac{\gamma(t) f(t) x_{*}}{\alpha(t)\left[\alpha(t)+\beta(t) x_{*}+\gamma(t) y^{*}\right]}\right. \\
&\left.\quad-\frac{\alpha(t) c(t)+c(t) \beta(t) x^{*}}{\alpha(t)\left[\alpha(t)+\beta(t) x_{*}+\gamma(t) y_{*}\right]}\right] \\
&>0.0117-0.001=0.0107 .
\end{aligned}
$$

Then all conditions of Theorem 25 are satisfied. By Theorem 25, system (46) has a unique positive almost periodic solution, which is globally attractive. This completes the proof.

\section{Conclusion}

In this paper, some sufficient conditions are established for the existence of positive almost periodic solution for a predator-prey system with Beddington-DeAngelis functional response on time scales by using the theory of exponential dichotomy on time scales and fixed point theory based on monotone operator. Further, the global attractivity of the almost periodic solution for the above continuous system is also investigated. The main results obtained in this paper are completely new even in case of the time scale $\mathbb{T}=\mathbb{R}$ or $\mathbb{Z}$. Besides, the method used in this paper may be used to study the positive almost periodic solution of many other biological models.

\section{Conflict of Interests}

The author declares that there is no conflict of interests regarding the publication of this paper.

\section{References}

[1] J. R. Beddington, "Mutual interference between parasites or predators and its effect on searching efficiency," Journal of Animal Ecology, vol. 44, pp. 331-340, 1975.

[2] D. L. DeAngelis, R. A. Goldstein, and R. V. O’Neill, "A model for trophic interaction," Ecology, vol. 56, no. 4, pp. 881-892, 1975.

[3] M. Fan and Y. Kuang, "Dynamics of a nonautonomous predator-prey system with the Beddington-DeAngelis functional response," Journal of Mathematical Analysis and Applications, vol. 295, no. 1, pp. 15-39, 2004.

[4] R. S. Cantrell and C. Cosner, "On the dynamics of predator-prey models with the Beddington-DeAngelis functional response," Journal of Mathematical Analysis and Applications, vol. 257, no. 1, pp. 206-222, 2001.

[5] R. S. Cantrell and C. Cosner, "Effects of domain size on the persistence of populations in a diffusive food-chain model with Beddington-DeAngelis functional response," Natural Resource Modeling, vol. 14, no. 3, pp. 335-367, 2001.

[6] T. Hwang, "Global analysis of the predator-prey system with Beddington-DeAngelis functional response," Journal of Mathematical Analysis and Applications, vol. 281, no. 1, pp. 395-401, 2003.

[7] J. Zhang and J. Wang, "Periodic solutions for discrete predator-prey systems with the Beddington-DeAngelis functional response," Applied Mathematics Letters, vol. 19, no. 12, pp. 1361-1366, 2006.

[8] J. Cui and Y. Takeuchi, "Permanence, extinction and periodic solution of predator-prey system with Beddington-DeAngelis functional response," Journal of Mathematical Analysis and Applications, vol. 317, no. 2, pp. 464-474, 2006.

[9] M. Bohner, M. Fan, and J. Zhang, "Existence of periodic solutions in predator-prey and competition dynamic systems," Nonlinear Analysis: Real World Applications, vol. 7, no. 5, pp. 1193-1204, 2006.

[10] M. Fazly and M. Hesaaraki, "Periodic solutions for predatorprey systems with Beddington-DeAngelis functional response on time scales," Nonlinear Analysis: Real World Applications, vol. 9, no. 3, pp. 1224-1235, 2008.

[11] S. Hilger, Ein Ma $\beta$ kettenkalkül mit anwendung auf zentrumsmannigfaltigkeiten [Ph.D. thesis], Universität Würzburg, 1988.

[12] M. Bohner and A. Peterson, Dynamic Equations on Time Scales, Birkhäauser, Boston, Mass, USA, 2001.

[13] E. R. Kaufmann and Y. N. Raffoul, "Periodic solutions for a neutral nonlinear dynamical equation on a time scale," Journal of Mathematical Analysis and Applications, vol. 319, no. 1, pp. 315-325, 2006.

[14] Y. Li and C. Wang, "Almost periodic functions on time scales and applications," Discrete Dynamics in Nature and Society, vol. 2011, Article ID 727068, 20 pages, 2011. 
[15] J. Zhang, M. Fan, and H. Zhu, "Existence and roughness of exponential dichotomies of linear dynamic equations on time scales," Computers \& Mathematics with Applications, vol. 59, no. 8, pp. 2658-2675, 2010.

[16] Y. Li and C. Wang, "Uniformly almost periodic functions and almost periodic solutions to dynamic equations on time scales," Abstract and Applied Analysis, vol. 2011, Article ID 341520, 22 pages, 2011.

[17] D. J. Guo, Nonlinear Functional Analysis, Shandong Science and Technology Press, Shandong, China, 2003 (Chinese).

[18] V. Kostrykin and A. Oleynik, "An intermediate value theorem for monotone operators in ordered Banach spaces," Fixed Point Theory and Applications, vol. 2012, article 211, 2012.

[19] R. P. Agarwal, M. Bohner, and P. Rehak, "Half-linear dynamic equations," in Nonlinear Analysis and Applications, vol. 1, pp. 157, Kluwer Academic Publishers, Dordercht, The Netherlands, 2003.

[20] B. Aulbach and L. Neidhart, "Integration on measure chains," in Proceedings of the $6 \mathrm{~h}$ International Conference on Difference Equations, pp. 239-252, CRC, Boca Raton, Fla, USA, 2004. 


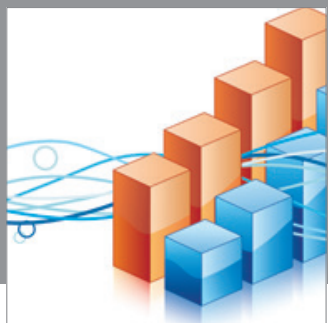

Advances in

Operations Research

mansans

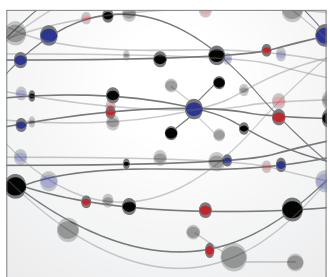

The Scientific World Journal
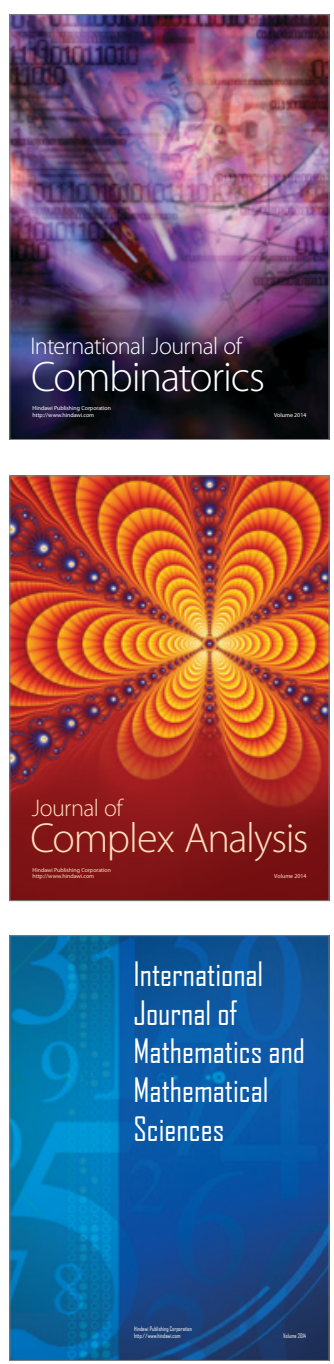
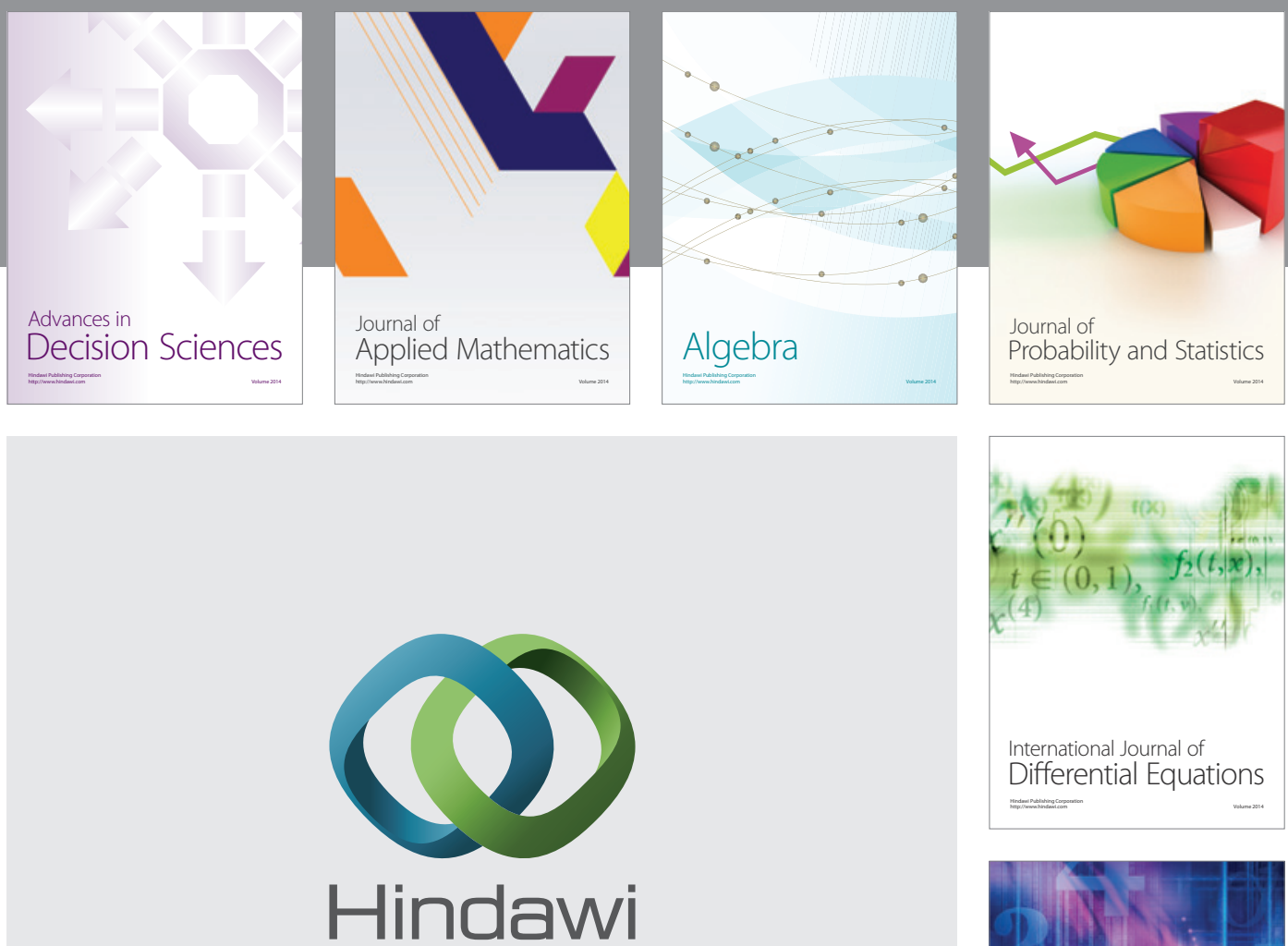

Submit your manuscripts at http://www.hindawi.com
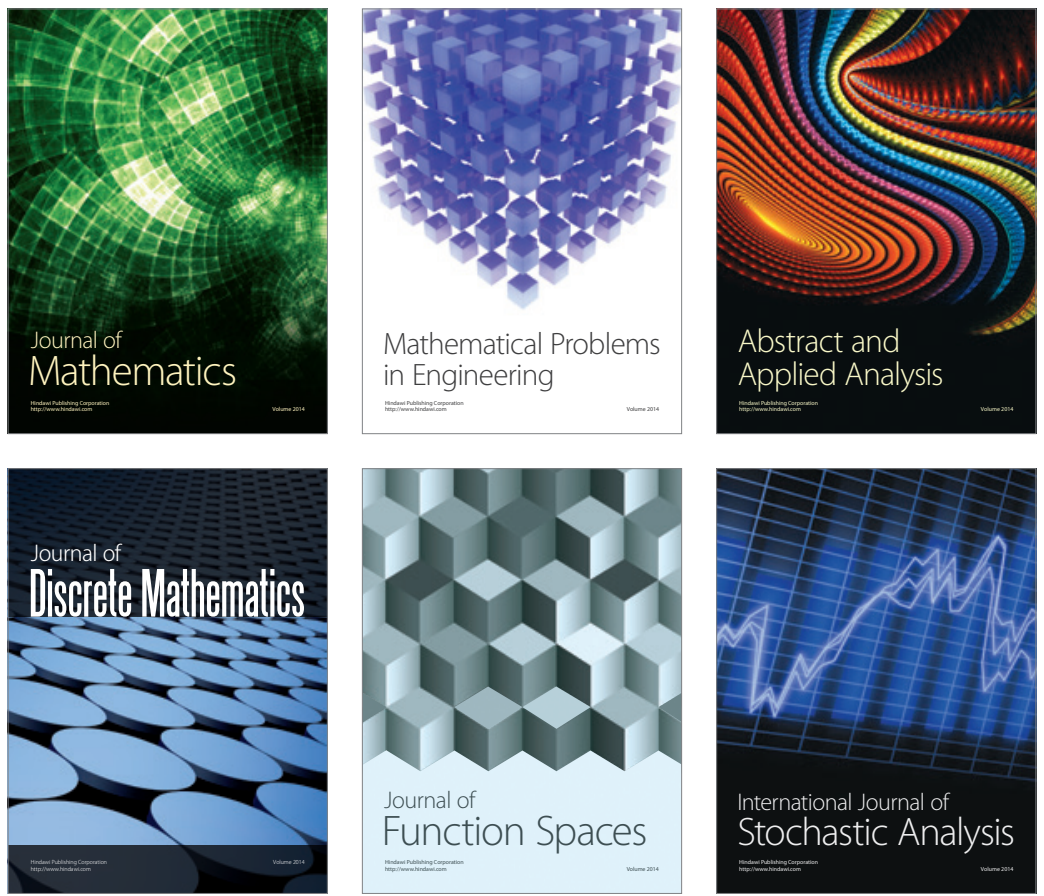

Journal of

Function Spaces

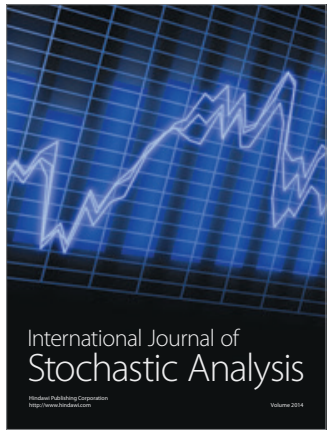

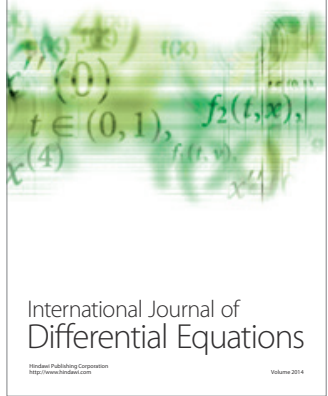
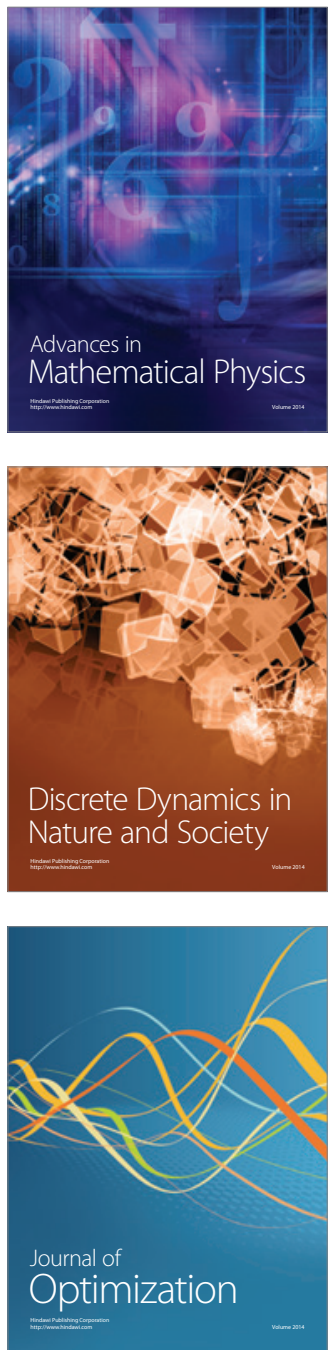